\title{
Gene Expression of GFP from jellyfish into fish.
}

\section{Omaima E. Khafagy ${ }^{1}$, Hawanem Sallam ${ }^{2}$, Inessa A. Zelenin ${ }^{3}$ and Alxander Zelenin ${ }^{4}$.}

1- Department of fish Resources and Aquaculture, Faculty of Environmental

Agricultural Sci., Suez Canal Univ., Egypt.

2- Department of Physical Chemistry, Faculty of Sci. Al-Azhar Univ., Egypt

3- Institute of Molecular Biology, Moscow, Academy of Sciences, Moscow.

4-Institute of Bioorganic Chemistry, Russian Academy of Sciences, Moscow.

\begin{abstract}
The green-fluorescent protein (GFP) of the jellyfish Aequorea victoria has recently been used as a universal reporter in a broad range of heterologous living cells and organisms. Fertilized eggs of the Carp (Cyprinus carpio) in the period of blastodisc formation and up to the fourth division of the cleavage were injected with two plasmids expressing the natural jellyfish GFP and synthetic engineered jellyfish (sGFP) using Microinjection method. Reverse transcription PCR products, cloning, sequencing, were analysed. Using universal transcription and translation enhancers with strong promoters, has been showed that GFP signals are detectable in transient expression systems of fish. The engineered GFP gene sequence gave higher GFP expression in fish than the natural jellyfish GFP sequence. This new tool can be used for studies of gene regulation, signal transduction, development and cell biology in animals and plants.
\end{abstract}

Key word: Gene expression, GFP, jellyfish, Cyprinus carpio, transgenic fish, fluorescent proteins,

\section{INTRODUCTION}

Marine biochemists labored in relative obscurity for 30 years before the jellyfish Aequorea victoria green fluorescent protein (GFP) gene was first cloned (Prasher et al.,1992) and transformed into the nematode Caenorhabditus elegans (Chalfie et al., 1994). Model organisms were the first to be transformed with GFP. After C. elegans came the fruitfly Drosophila melanogaster (Yeh,1995) followed by mammalian cell lines (Pines, 1995) and yeast (Niedenthal,1996). Larger organisms such as plants (Stewart, 2001), mouse (Okabe, 1997), zebrafish (Ju, 1999) and the frog Xenopus laevis (MarshArmstrong, 1999) were transformed with GFP variants yielding visible green fluorescence.

FPs quickly transcended science to be used in ornamental fish and even iconic works of art. The most famous of these is 'Alba', the GFP bunny commissioned by the artist Eduardo Kac. The erstwhile albino rabbit was engineered with GFP to yield a striking fluorescent phenotype, which was a 
focal point of the Eighth Day art exhibit, where art and biotechnology intersected (Prasher et al.,1992).

The transgenic rhesus monkey 'ANDi (Chan, 2001) was also iconic (at least from a new perspective) not because it had a macroscopically fluorescent body, but simply because it was the first reported GFP transgenic primate obviously reporters and the public had a closer taxonomic identity with the monkey than the bunny. The most successful commercial ornamental transgenic organism to date has been 'GloFish (www.glofish.com), marketed by Yorktown Technologies.

The cloning of the jellyfish green fluorescent protein (GFP) provided a protein expression tag that can be used to reveal the subcellular localization of labeled proteins in their natural environment within the living cell. The mutagenesis of GFP has yielded proteins with different spectral properties, and some of these different-color fluorescent proteins can be used in combination to monitor the behavior of more than one protein in the same living cell (Richard et al., 2001).

GFP was discovered more than 40 years ago (Johnson et al., 1962), but it was not until 1994, after cloning (Prasher et al., 1992) and successful heterologous expression of the GFP gene (Gene Bank accession no. U17997) (Chalfie et al., 1994), that this protein attracted attention. GFP and its mutants soon became popular tools for cell and molecular biology and during the past few years, the great spectral and phylogenetic diversity of GFP-like proteins has been characterized in marine organisms. Furthermore, several useful mutant variants of FPs have been generated. Consequently, a panel of fluorescent proteins is now available that covers almost the whole visible spectrum, each possessing different biochemical characteristics.

The green-fluorescent protein (GFP) of the jellyfish Aequorea victoria has recently been used as a universal reporter in a broad range of heterologous living cells and organisms (Wan-ling et al., 1996). Fluorescent intensity is required for GFP to be useful as a marker in intact organisms.

Coupled with electronic devices for visualizing and quantifying FPs, recently cloned FP genes might be useful for the ecological monitoring of transgenic organisms in the environment (Stewart, 2006).

\section{MATERIALS AND METHODS}

\section{Plasmid constructions}

The construction of the fish expression vector with a strong and constitutive promoter 35SC4PPDK has been described (Sheen et al.,1995). The creation of sGFP is reported separately (Haas et al.,1996), sGFP(S65T) was generated by PCR-based site-directed mutagenesis, using two flanking primers: 5-GCGGATCCATGGTGAGCAAG-3' and 5'GGGCGGCCGCTTTACTTGTA-3' and two overlapping mutagenesis primers: 
5' - GTGACCACCTTCACCTACGGCGTGCAG-3' and 5'-CTGCACGCCGTAGGGAAGGTGGTCAC-3'.GFP. and sGFP (S65T) were amplified using the same flanking PCR primers and inserted into the expression vector between BamHI and SmaI sites. Two clones were picked from each construction for initial evaluation. The ArabidopsisCAB2 (Anderson and Kay, 1995) and RD29A (Yamaguchi-Shinozaki and Shinozaki, 1994) Promoters were obtained by PCR and fused to the NcoI site at the 5'of sGFP(S65T). Two clones were selected for initial evaluation by transient expression analysis. The primers used were :

5'-TGGACTAGAGATTGCCACGTA-3' and

5'-AGGAGAGAGCCATGGTTGAGGC-GGCCAT-3' for the AtCAB2 promoter and 5'-GACCGACTACTAAT-AATAGTAAGT-3' and

5'-TGTTTGATCCATGGTCCACCGATTTTT-3'. for the AtRD29A promoter. The constructs used for microinjection carried the $35 \mathrm{SV}$ regulatory sequence instead of the 35SC4PPDK promoter (Sheen J et al.,1995). The NLS of SV40 (Van der Krol and Chua, 1991) was synthesized :

(5'-TCGACCATGGCTCCAAAGAAGAAGAGAAAGGT-3' and

5'-ATGACCTTTCTCTTCTTCTTTGGAGCCATGG-3'), annealed and kinased before insertion into the SalI and NcoI site of the 35V-sGFP(S65T) plasmid.

Two similar constructs were generated by inserting GFP, sGFP(S65T) into fish eggs expression vector with a strong, constitutive promoter (35SC4PPDK) (Sheen et al.,1995). Plasmid DNA introduced into fertilized fish eggs by microinjection has been done in Moscow (Russia) by Inessa Zelenin and Alxander Zelenin (Institute of Bioorganic Chemistry, and Institute of Molecular Biology, Moscow, Russian Academy of Sciences).

There are different methods available for the transient expression of GFPfusion proteins in living cells. The efficiency of these methods can be very dependent on the cell type being used (Richard et al., 2001). For such studies microinjection of fertilized fish eggs has been used.

\section{Genomic DNA Isolation:}

Genomic DNA was extracted from samples of the Carp (Cyprinus carpio) then, injected in Moscow.by Inassa Alxanrrevna following the method of Maniatis et al. (1982), with some modifications. Samples were lysed in $50 \mathrm{ml}$ of $50 \mathrm{mml} / 1$ Tris buffer ( $\mathrm{pH} \mathrm{8.0)} \mathrm{had} \mathrm{been} \mathrm{sent} \mathrm{frozen} \mathrm{to} \mathrm{Cairo.} \mathrm{The} \mathrm{genomic} \mathrm{DNA}$

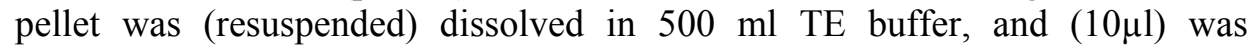
aliquoted for PCR concentration determined.

\section{PCR amplification :}

Samples were used directly for PCR (Shears et al., 1991) with different primers. The PCR products were run in $0.8 \%$ agarose gels by electrophoresis, Amplification Conditions and electrophoresis conditions were carried out according to Williams et al .(1990).

Approximately $15 \mu \mathrm{lpf}$ amplification products were separated on $1.5 \%$ agarose until the marker bands give a good resolution. The gel was immediately 
photographed using Polaroid camera. $15 \mu \mathrm{l}$ of DNA implied product was loaded in each well and $1 \mathrm{~Kb}$ (Sigma) DNA marker (23,9.4,7.4,6.6,5.3, 4.4, 2.3,1.9,1.6 Kbp) mix was used as standard DNA (Fig.1). Similarly, other DNA marker $(340,330,310,300,300,281,271 \mathrm{bp})$ mix was kindly supplied by the Russian investigates and used (Figs. $2 \mathrm{~A}, \mathrm{~B})$.
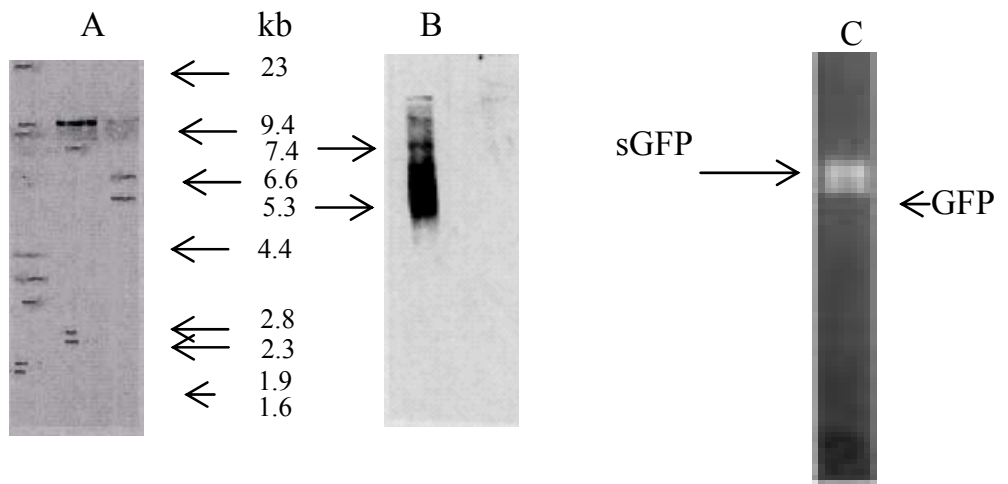

Fig. (1) :DNA Size markers are indicated on the right $(A)$ and the left $(B)$.

A band of approximately $10.5 \mathrm{~kb}$, additional bands of 7,5.5,4.4,2.8,2,1.9,1.5 are detectable as shown.

C: Engineered GFP gives faster and brighter fluorescent signal.

A

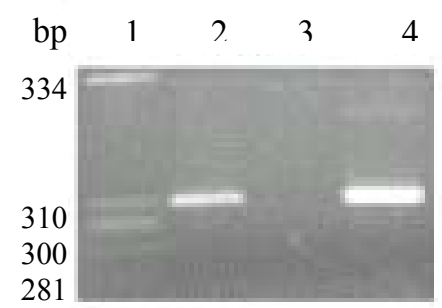

B

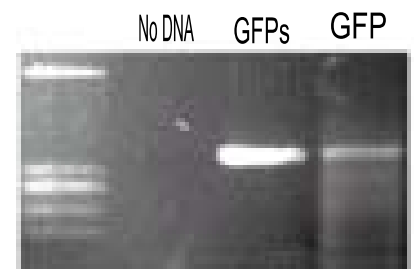

Fig.2: Engineered GFP gave faster and brighter fluorescent signals

(A)

1. (Marker).

2. (wild type of GFP).

3. (without DNA).

4. (Transfected cell with sGFP).
(B)

1. (Marker).

2. (without DNA).

3. (wild type of GFP).

4 (Transfected cell with sGFP).

\section{SDS-PAGE electrophoresis:}

Extraction of isozyme, electrophoresis conditions, gel preparation, staining and distaining were conducted according to Tanksley and Orton (1983). Gel electrophoresis for fish-stock identification. 
The SDS-PAGE gel were used in the separation. Following the separation, the gel was removed, using solution of Coomassie Brilliant Blue and destained according to the procedures used by Williams, et al., (1998).

Standard molecular weight marker protein (wide range) obtained from Sigma Chemical Company, was applied as sample to the gel. method of (Laemmli, 1970). Standard molecular. with some modifications. For the $0.85 \%$ $\mathrm{NaCl}$ and $70 \%$ ethanol soluble proteins 20 and $40 \mu \mathrm{l}$ were added to SDS sample buffer, $(\mathrm{pH}$ 8.8). Control wells were loaded with standard protein markers (100, 92,66,46 and $30 \mathrm{KDa})$ (Fig.3) and loaded with standard protein markers (340 and $330 \mathrm{KDa}$ ) (Fig.4). The banding patterns of individual sample were compared between original GFP and sGFP.

Untransfected control and transfected fish were labeled. Total proteins were solublized in protein loading buffer and separated by $12.5 \%$ SDS-PAGE.

\section{Analysis of organelle targeting in fish}

All gels resulted from protein and DNA electrophoresis, were scanned and analysed. The output results involved both different hierarchical pair distance (UPGAMA) and constructed dendrogram.

\section{RESULTS}

A mutation of replacement of the serine position 65 with a threonine (S65T) has been shown, faster formation and slower. This change was also introduced into sGFP by site-directed mutagenesis, to create sGFP (S65T).

This early detection of sGFP (S65T) was due mainly to the enhanced fluorescent signal and rapid formation induced by the S65T mutation, as shown in E. coli Heim et al.,1995. As GFP and sGFP have identical amino-acid sequences, the brighter signal from sGFP is likely to be due to a higher level of protein synthesis caused by optimal codon usage, as demonstrated by Haas et al. in a preceding paper (Haas et al.,1996). As shown in Figs. (1 - 4), the amounts of newly synthesized GFP and sGFP (S65T) were similar and easily detectable without purification, whereas the amount of native GFP was lower as agreement with Wan-ling, et al.,1996. Similar results were obtained on different m RNA preparations and by hybridization experiments with a $360 \mathrm{bp}$ PCR probe corresponding the neucleotides (1380-1620) of the plasmid insert by Inessa Zelenin, 2006) who typically found that approximately $40 \%$ of the cells expressed the fusion proteins as determined by fluorescence microscopy as has been reported in late researches.

It has been shown that the engineered GFP gene sequence, with the favored codons of highly expressed proteins, gave higher GFP expression in fish cells than the original jellyfish GFP sequence (Figs. 3 and 4). It has shown that this modified GFP has a broad use in various transient expression systems, and allows the easy detection of weak promoter activity, visualization of protein targeting into the nucleus and analysis of signal transduction pathways in living single cells and in transgenic animals (Wan-ling, et al., 1996). 


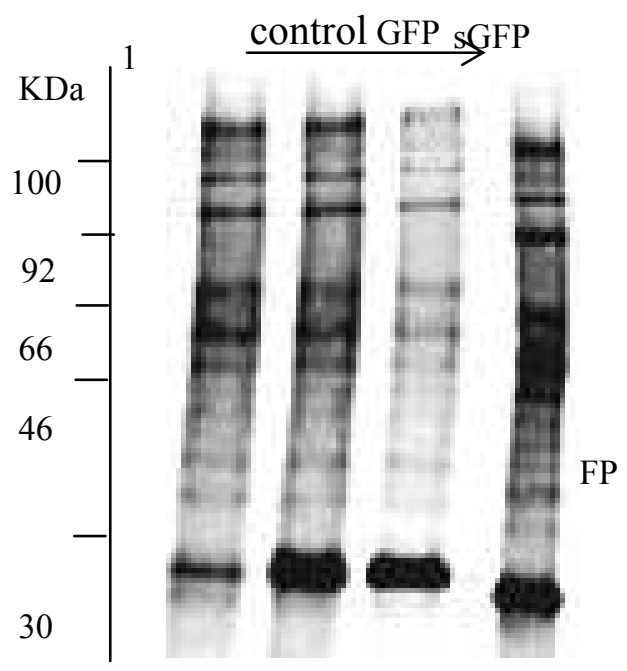

Fig. 3. Synthetic GFP gives a high level of protein expression.

Untransfected control and transfected cell were labeled. Total proteins were solublized in protein loading buffer and separated by SDS-PAGE.

Engineered GFP gives faster and brighter fluorescent signals.

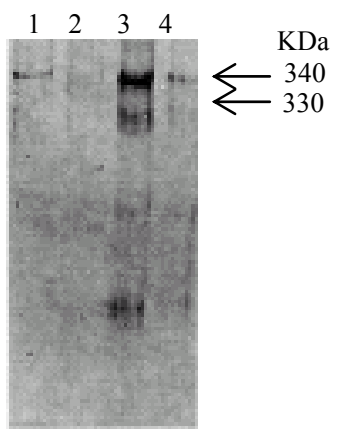

Fig. (4): Line1Normal GFP, 2 N0 cell ,3 transfected cell with sGFP and 4 marker.

Engineered GFP gives faster and brighter fluorescent signals (Fig.2). Fish cells were injected with two plasmids expressing; the jellyfish GFP and sGFPS65T. Synthetic GFP gives a high level of protein expression. Cells respond to environmental cues by modifying protein complexes in the nucleus to produce a change in the pattern of gene expression. 


\section{DISCUSSION}

Higher levels of GFP expression are still important to broaden its applications in mammals and higher plants. By combining optimal codon usage (Haas et al.,1996) and the S65T mutation1 (Heim et al.,1995) we have found that sGFP (S65T) gives higher expression levels, faster formation and more enhanced fluorescent emission in fish cells, that agreed with Wan-ling, et al., (1996).

A universal transcription or translation enhancer was essential to use the original jellyfish GFP in fish cells (Sheen et al.,1995; Galbraith. et al.,1995). It has been shown here that GFP and sGFP (S65T) can be used to detect the activity of weaker promoters. The expression of GFP and sGFP (S65T) also provides a rapid, simple and non-destructive assessment of transient transfection and stable transformation efficiency (Millar et al., 1992).

The green fluorescent protein (GFP) from the jellyfish Aequoria victoria was cloned by Prasher et al. (1992). The expression of GFP either alone or as a fusion to other proteins in a variety of cell types and in transgenic organisms has proven its utility as an in vivo reporter (Chalfie et al.,1994; Xian et al.,1999). The relatively low molecular weight of GFP makes it ideal as a fluorescent tag in fusion proteins. Protein sorting, traffic, localization, movement can be followed in real time at high resolution, especially with confocal microscopes (Prasher et al.,1992; Wang and Hazelrigg, 1994).

Fusion proteins with various fluorescent tags also provide a new way to detect protein-protein interactions or changes in protein conformation in vivo.

The sGFP(S65T) reporter is a convenient tool for further studies at the single-cell level, and can label cells for functional and physiological analysis of co-transfected genes. It also has potential as a vital marker for enhancer and gene trap screening, investigating recombination and transposition events, fate mapping or cell lineage analysis, tracing chromosome or gene segregation, genetic and molecular mapping, and mutant selection. (Wan-ling, et al., 1996).

Serendipitously, the sequence at the cryptic splice donor site AAAGGTATTGATTTTAAA was changed to AAgGGcATcGATTTcAAg during the synthesis of sGFP with favored codons, and the cryptic intron in the coding region (400 - 483) of GFP (Haseloff and Amos, 1995) is therefore eliminated in GFP and sGFP (S65T) (Fig. 5).Expression of engineered GFP in fish cells as each GFP molecule represents, high level expression is important to give good fluorescent signals (Wan-ling, et al., 1996). 


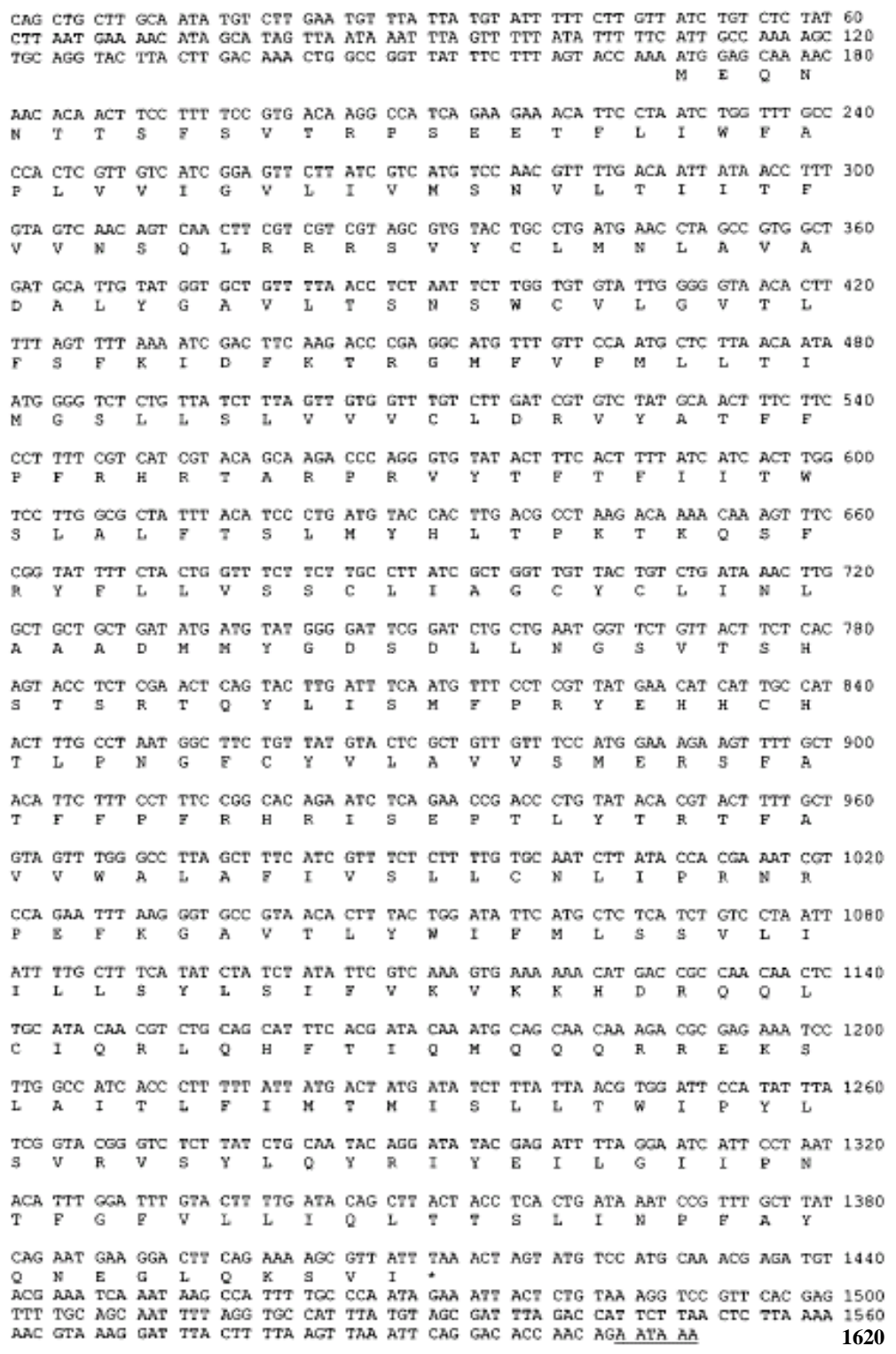

Fig. (5 ).Nucleotide and deduced amino acid sequences of the jellyfish receptor. The nucleotide of the genomic clone is represented. The putative olyadenylation sequence AATAAA is underlined. The amino acids within the predicted open reading frame are also shown.

Aequorea victoria, and its mutant variants, are the only fully genetically encoded fluorescent probes available and they have proved to be excellent tools for labeling living specimens (Dmitriy et al. 2005).

Today, fluorescent labeling is of paramount importance to biological studies and a great number of chemical dyes are used extensively to label 
biological specimens; however, these dyes must be added exogenously, which can be incompatible with living systems (Dmitriy et al., 2005).

It has been shown that SGFP and SGFP(S65T) are versatile and sensitive reporters in transient expression using animal cells and in transgenic fish and mammals . These new tools can be used for studies of gene regulation, signal transduction, development and cell biology in fish (Dmitriy et al., 2005).

The modified GFP is a simple and economical new tool for the direct visualization of promoter activities with a broad range of strength and cell specificity. It can be used to measure dynamic responses of signal transduction pathways, transfection efficiency and subcellular localization of chimeric proteins, and should be suitable for many other applications in genetically modified living cells (Wan-ling, et al., 1996).

The data also suggest that the codon usage effect might be universal, allowing the design of recombinant proteins with high expression efficiency in evolutionarily distant species such as humans and maize.

The use of different-color fluorescent proteins in combination with FRET offers the opportunity to study the complex behavior of key regulatory proteins in their natural environment within the living cell.

This work can serve as a powerful tool, therefore, for elucidating the mechanisms of gene regulation and signal transduction in fish (Wan-ling, et al., 1996).

The $\mathrm{sGFP}(\mathrm{S} 65 \mathrm{~T})$ as a reporter for a weak promoter, in combination with the $\operatorname{sGFP}(\mathrm{S} 65 \mathrm{~T})$ marker, where cells can be used conveniently to study signal transduction and cell-cycle regulation in fish cells.

\section{REFERENCES}

Chalfie, M..; Tu, Y.; Euskirchen, G.; Ward, W. W. and Prasher, D. C. (1994). Green fluorescent protein as a marker for gene expression. Science, 263: 802-805.

Chan, A.W.S. (2001). Transgenic monkeys produced by retroviral gene transfer into mature oocytes. Science 291: 309-312.

Dmitriy, M.; Chudakov, S. and Konstantin A. (2005).Fluorescent proteins as a toolkit for in vivo imaging. TRENDS in Biotechnology, 23(12):605-613.

Haas, J.; Park, E-C. and Seed, B1(996). Codon usage limitation in the expression of HIV-1 envelope glycoprotein. Curr. Biol., 6:315-324.

Haseloff, J. and Amos, B. (1995). GFP in plants. Trends Genet, 11:328-329. 
Heim, R.; Cubitt, A. B. and Tsien, R.Y. (1995). Improved green fluorescence. Nature, 373:663-664.

Ju, B. (1999). Faithful expression of green fluorescent protein (GFP) in transgenic zebrafish embryos under control of zebrafish gene promoters. Dev. Genet., 25: 158-167.

Laemmli, U. K. (1970). Cleavage of structural proteins during the assembly of the head of bacteriophage T4. Nature., 227:680-258.

Maniatis, T.; Fritsch, E. F. and Sambrook, J. (1982). Molecular Cloning : A Laboratory Manual.(Cold Spring Harbor Laboratory, Cold Spring Harbor, New York..

Marsh-Armstrong, N. (1999). Germ-line transmission of transgenes in Xenopus laevis. Proc. Natl. Acad. Sci. U. S. A., 96:14389-14393.

Millar, A. J.; Short, S. R.; Hiratsuka, K.; Chua, N-H. and Kay, SA.(1992). Firefly luciferase as a reporter of regulated gene expression in higher plants. Plant. Mol. Biol. Rep., 10:324-337.

Niedenthal, R. K. (1996). Green fluorescent protein as a marker for gene expression and subcellular localization in budding yeast. Yeast 12: 773786.

Okabe, M. (1997). Green fluorescent protein as a novel vital marker in transgenic mouse. J. Reprod. Dev., 43: 19-25.

Pines, J. (1995). GFP in mammalian cells. Trends Genet., 11:326-327.

Prasher, D. C.; Eckenrode, V. K.; Ward, W. W.; Prendergast, F. G.and Cormier, M. J. (1992). Primary structure of the Aequorea victoria greenfluorescent protein. Gene 111: 229-233.

Richard, N.; Day, A. P. and Fred, S. (2001). Fluorescence Resonance Energy Transfer Microscopy of Localized Protein Interactions in the Living Cell Nucleus., Methods, 25: 4-18.

Shears, M. A.; Fletcher, G. L.; Hew, C. L.; Gauthier, S. and Davies, P. L. (1991). Transfer, expression and stable inheritance of antifreeze protein genes in Atlantic salmon (Salmo salar). Mol Marine Biol Biotechnology, 1: 58-63. 
Sheen, J.; Hwang, S.; Niwa, Y.; Kobayashi, H. and Galbraith,D.W. (1995). Greenfluorescent protein as a new vital marker in plant cells. Plant J., 8:777-784.

Stewart, C. and Neal, Jr. (2006). Go with the glow: fluorescent proteins to light transgenic organisms. TRENDS in Biotechnology, 24 (4C.)

Stewart, C. and Neal, Jr. (2001). The utility of green fluorescent protein intransgenic plants. Plant Cell Rep., 20:376-382.

Tanksley, S. and Orton, T. (1983). Isozymes in plant genetic and breeding . Part (B). Elsevier Science Bub b.V. Amesterdam.

Van der Krol AR and Chua N-H (1991). The basic domain of plant B-ZIP proteins facilitates import of a reporter protein into plant nuclei. Plant Cell, 3:667-675.

Wang, S. and Hazelrigg, T. (1994). Implications for bcd mRAN localization from spatial distribution of exu protein in Drosophila oogenesis. Nature, 369:400-403.

Wan-ling, C.; Yasuo, N.; Weike, Z.; Takanori, H.; Hirokazu, K. and Jen, S. (1996). Engineered GFP as a vital reporter in plants.. Current Biology, 6 (3):325-330.

Williams, D. J.; Kazianis, S. and Walter, R. B. (1998). Use of Random Amplified Polymorphic DNA (RAPD) for identification of large mouth bass subspecies and their intergrades.Transactions of the American Fisheries Society, 127(5)825.

Williams, J. G; Kubelik, A. R.; Livak, K. J.; Rafalski, J. A. and Tingy, S. V. (1990). DNA polymorphism amplified by arbitrary primers are used as genetic markers. Nucleic Asid Res.,18:6531-6535.

Xian, M.; Honbo, N.; Zhang, J.; Liew, C. C.; Karliner, J. S. and Lau, Y. F. (999). J. Mol. Cell Cardiol., 31: 2155-2165.

Yamaguchi-Shinozaki K and Shinozaki K (1994). A novel cis-acting element in an Arabidopsis gene is involved in responsiveness to drought, lowtemperature, or high-salt stress. Plant Cell, 6:251-264.

Yeh, E. (1995). Green fluorescent protein as a vital marker and reporter of gene expression in Drosophila. Proc. Natl. Acad. Sci. U.S.A., 92: 7036-7040. 